\title{
Evaluation of splenic perfusion and spleen size using dynamic-CT: usefulness in assessing degree of liver \\ fibrosis
}

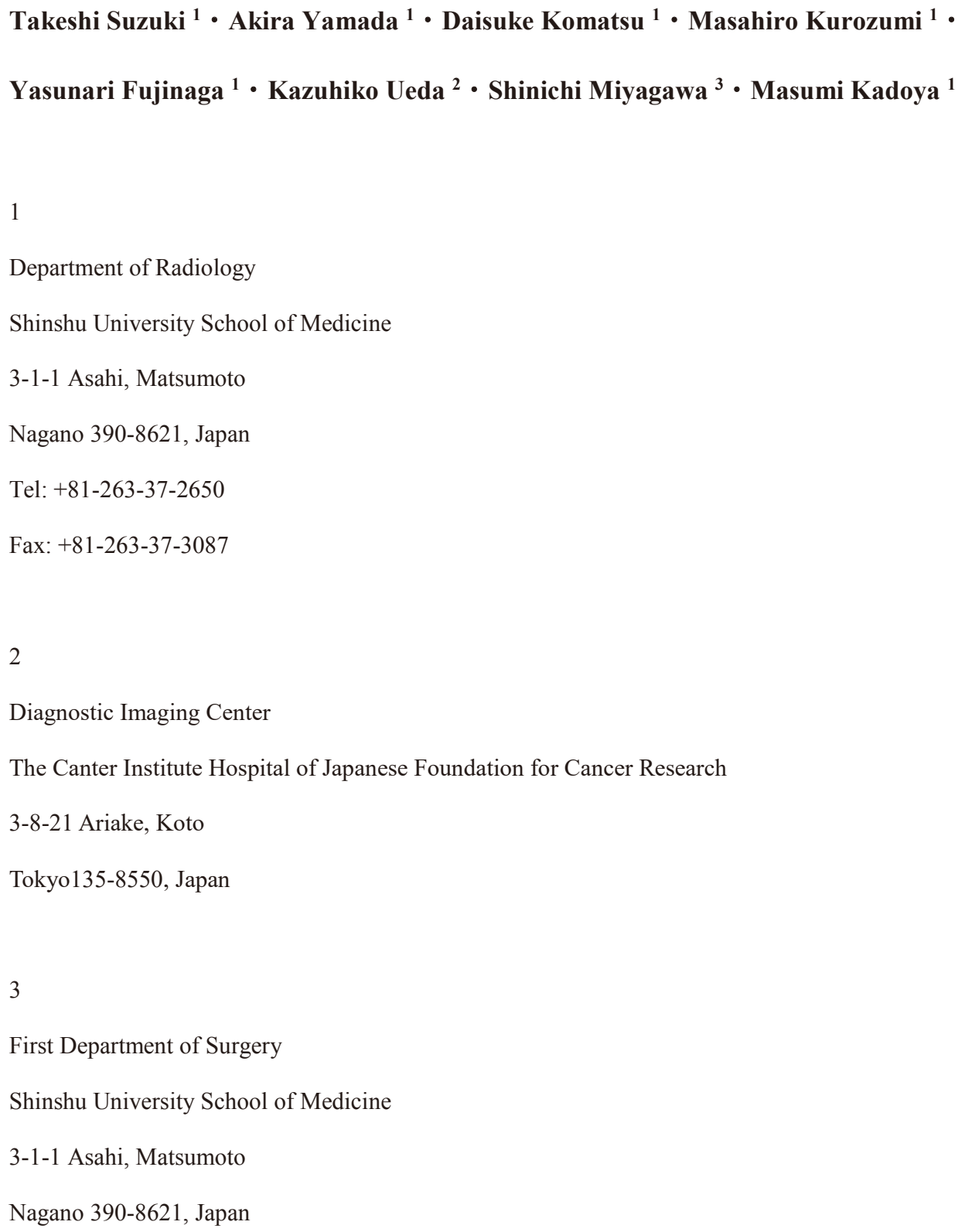


Aim: To enhance the usefulness of splenic perfusion evaluated by means of dynamic CT and of spleen size in assessing the degree of liver fibrosis.

Methods: We retrospectively studied 133 patients who had undergone dynamic CT before hepatectomy. Fibrosis was histologically established in all. First we calculated splenic perfusion parameters $\mathrm{K}_{1}$ (inflow rate constant), $1 / \mathrm{k}_{2}$ (Mean Transit Time: MTT), and $\mathrm{K}_{1} / \mathrm{k}_{2}$ (distribution volume: $\mathrm{V}_{\mathrm{d}}$ ), using compartment model analysis. Then we compared the stage of fibrosis with splenic perfusion and spleen size (long axis: R), using the Kruskal-Wallis test and multiple comparisons. After that, we assessed the diagnostic accuracy of the combination of splenic perfusion, spleen size, age, gender, the presence or absence of hepatitis B viral (HBV) infection, and that of hepatitis $\mathrm{C}$ viral (HCV) infection, in detecting liver fibrosis, using stepwise regression and receiver operating characteristic (ROC) analysis.

Results: Significant differences $(\mathrm{p}<0.05)$ in MTT were observed in comparisons between F0 and F4, between F1 and F4, and between F2 and F4. Significant differences $(\mathrm{p}<0.05)$ in $\mathrm{R}$ were observed in comparisons between F0 and F4, and between F1 and F4. Considering the presence or absence of HBV/HCV infection along with MTT and R, the areas under the ROC curves (AUROC) were 0.89 for $\geqq$ $\mathrm{F} 1,0.83$ for $\geqq \mathrm{F} 2,0.82$ for $\geqq \mathrm{F} 3$, and 0.82 for $\mathrm{F} 4$.

Conclusion: Splenic MTT and spleen size are helpful in assessing liver fibrosis.

\section{Keywords}

Liver fibrosis $\cdot$ Multidetector Computed Tomography $\cdot$ Perfusion $\cdot$ Portal hypertension $\cdot$ Spleen

\section{Introduction}

It is useful to assess the degree of liver fibrosis before making decisions or predicting disease outcomes in chronic liver disease ${ }^{1}{ }^{2}$. Liver fibrosis can currently be evaluated without biopsy using serum fibrosis markers and liver elastography ${ }^{1,3}$. However, under certain conditions these methods produce divergent results, and biopsy becomes necessary ${ }^{3}$. Finding another non-invasive method will reduce this necessity.

To some extent, the amount of splenic perfusion indicates the degree of liver fibrosis. It has been shown by means of the maximum slope method that normal spleens and spleens associated with liver cirrhosis display different amounts of perfusion ${ }^{4-6}$. Sauter et al ${ }^{7}$ demonstrated by means of compartment model analysis 
that Mean Transit Time (MTT) was longer in spleens with cirrhosis than in normal spleens. However, there have been no studies comparing splenic perfusion among patients with various intermediate stages of liver fibrosis. Thus, the usefulness of splenic perfusion in assessing the degree of liver fibrosis has not been elucidated.

Splenomegaly is often used radiologically as an indicator of cirrhosis. It has been shown that portal venous pressure positively correlates with spleen size ${ }^{8-12}$. Therefore, we evaluated the usefulness of splenic perfusion and spleen size in assessing the degree of liver fibrosis. The aim of this study was to pinpoint the extent to which splenic perfusion indicates the degree of liver fibrosis and to enhance the usefulness of splenic perfusion evaluated by means of dynamic computed tomography (CT), and of spleen size, in assessing the degree of liver fibrosis.

\section{Materials and methods}

Patient characteristics

This retrospective study was approved by our Institutional Review Board, and written informed consent was obtained. Patient characteristics are shown in Table 1. We listed 136 consecutive patients from 2008 to 2013 who had undergone multiphasic intravenous contrast-enhanced dynamic CT within 30 days before hepatectomy. Makuuchi's criteria ${ }^{13}$ were used in determining whether or not each patient should undergo hepatectomy. Fibrosis was histologically established in all of the patients by means of surgical samples; we extracted the stage of fibrosis described in the pathological reports. Three of the 136 patients were excluded from this study, two because they had undergone splenectomies, and one because the aorta was too small in diameter (region of interest [ROI] in the aorta $<200 \mathrm{~mm}^{2}$ ), so we studied 133 patients ( 87 men and 46 women; age range, 37-83 years; median age, 68 years). The median time interval between CT and hepatectomy was 14 days. The respective numbers of patients according to stage of fibrosis were as follows: F0 (absent fibrosis):43; F1 (portal fibrosis):17; F2 (portal fibrosis with few septa):30; F3 (septal fibrosis):16; F4 (cirrhosis):27, as shown in Table 1. There were 37 patients with hepatocellular carcinoma (HCC) secondary to hepatitis $\mathrm{C}$ viral (HCV) infection, and 22 with $\mathrm{HCC}$ secondary to hepatitis B viral 
(HBV) infection. There was $1 \mathrm{HCV}$-infected patient with cholangiocellular carcinoma. Neither HBV infection nor $\mathrm{HCV}$ infection were present in the remaining 73 patients. Of these 73 patients, there were 21 with HCC related to other causes, including 11 with alcoholic hepatitis, 2 with nonalcoholic steatohepatitis (NASH), 8 where the cause was unknown (we defined "unknown" as patients without an obvious history of alcohol abuse or steatosis, except for patients who matched the criteria of NASH and patients with clinically suspected NASH), 23 with metastatic liver tumors, 16 with cholangiocellular carcinoma, 6 with perihilar cholangiocarcinoma, 2 with gallbladder carcinoma, 1 with xanthogranulomatous cholecystitis, 1 with a hepatic cyst, and 3 transplant recipients (NASH:1; familial amyloid polyneuropathy:1; exacerbation of alcoholic hepatitis:1).

CT protocol

The protocol of dynamic contrast-enhanced CT is shown in Table 2. We used a 64-row CT scanner, the Light Speed VCT (GE Healthcare, Little Chalfont, UK). Pre-contrast phase and 9 phases after intravenous contrast agent injection were scanned: 7 at 6-second intervals beginning at 22 seconds after injection, and 1 each at 90 and 210 seconds after injection. Scan parameters were as follows: the range was caudal $25 \mathrm{~cm}$ from the upper level of the diaphragm; the tube voltage was $120 \mathrm{kVp}$; the tube current was $300 \mathrm{~mA}\left(2^{\text {nd }}\right.$ through $8^{\text {th }}$, and $10^{\text {th }}$, phases $)$, or $500 \mathrm{~mA}\left(1^{\text {st }}\right.$ and $9^{\text {th }}$ phases $)$; the matrix was $512 \times 512$ pixels; the field of view was $320 \times 320 \mathrm{~mm}$; the size of collimation was $0.625 \mathrm{~mm}$; the reconstruction thickness was $2.5 \mathrm{~mm}$. The median (interquartile range) effective dose was $48.9 \mathrm{mSv}$ (48.2-48.9). A nonionic iodinated contrast agent (Iopamiron $370 \mathrm{mg} / \mathrm{mL}$, Bayer Healthcare, Berlin, Germany) was administered intravenously through a 22-gauge catheter in the median cubital vein. The total dose was $100 \mathrm{~mL}$, and the rate of injection was 3 $\mathrm{mL} / \mathrm{s}$. This CT protocol was adopted, instead of angiography, CT during arterioportography, or CT during hepatic arteriography, for preoperative mapping of vascular anatomy and detection of small lesions.

Image analysis and statistics

Two radiologists (TS and AY), with 2 and 16 years of experience respectively, set ROIs in consensus as large as possible, located at the spleen $\left[\mathrm{C}_{\mathrm{s}}(\mathrm{t})\right]$ and at the aorta $\left[\mathrm{C}_{\mathrm{a}}(\mathrm{t})\right]$ in the same slice, as shown in Figure 
1, and obtained time-attenuation data generated from the ROIs. The radiologists are among the authors, but they set the ROIs without knowing the degree of fibrosis in each patient. The minimum area of the ROIs was restricted to $300 \mathrm{~mm}^{2}$ at the spleen and $200 \mathrm{~mm}^{2}$ at the aorta. The concentration of iodine was directly proportional to the $\mathrm{CT}$ value ${ }^{14}$, and changes in the $\mathrm{CT}$ value in the target organ were expressed by the differential equation $\mathrm{dC}_{\mathrm{s}}(\mathrm{t}) / \mathrm{dt}=\mathrm{K}_{1} \mathrm{C}_{\mathrm{a}}(\mathrm{t}-\tau)-\mathrm{k}_{2} \mathrm{C}_{\mathrm{s}}(\mathrm{t})$ in the compartment model. Then we calculated the perfusion parameters of the spleen $-\mathrm{K}_{1}$ (inflow rate constant), $1 / \mathrm{k}_{2}$ (Mean Transit Time: MTT), and $\mathrm{K}_{1} / \mathrm{k}_{2}$ (distribution volume: $\mathrm{V}_{\mathrm{d}}$ ) - using the non-linear least squares method. In addition, we measured the long axis of the spleen (a classic fibrosis marker) and called it R. Then we compared the stages of fibrosis with splenic perfusion and spleen size using the Kruskal-Wallis test and multiple comparisons (Steel-Dwass test). After that, we assessed the diagnostic accuracy of the combination of splenic perfusion, spleen size, age, gender, the presence or absence of HBV infection, and that of HCV infection, in detecting liver fibrosis, using stepwise regression and receiver operating characteristic (ROC) analysis. In stepwise regression, the selection criterion was the following: the program added the term to the model if $\mathrm{p}$ was smaller than 0.05 , and removed the term from the model if $\mathrm{p}$ was larger than 0.10 . The image analysis and the statistical analysis were performed using MATLAB software (Mathworks, Natick, US).

\section{Results}

The median (interquartile range) $\mathrm{K}_{1}$ at F0, F1, F2, F3, and F4 were $0.0339 \mathrm{~s}^{-1}$ (0.0291 to 0.0374$), 0.0321 \mathrm{~s}^{-}$

${ }^{1}$ ( 0.0275 to 0.0355$), 0.0317 \mathrm{~s}^{-1}(0.0290$ to 0.0359$), 0.0295 \mathrm{~s}^{-1}(0.0260$ to 0.0348$)$, and $0.0243 \mathrm{~s}^{-1}$ (0.0187 to 0.0322), respectively. The median (interquartile range) MTT at F0, F1, F2, F3, and F4 were $16.4 \mathrm{~s}$ (14.8 to 18.9), $16.7 \mathrm{~s}$ (14.9 to 18.5$), 17.0 \mathrm{~s}$ (15.2 to 18.4$), 18.4 \mathrm{~s}$ (15.9 to 19.0), and $21.0 \mathrm{~s}$ (17.5 to 30.6), respectively. The median (interquartile range) $\mathrm{V}_{\mathrm{d}}$ at F0, F1, F2, F3, and F4 were $56.0 \%$ (52.8 to 59.7 ), $56.6 \%$ (50.1 to 58.5 ), $55.2 \%$ (52.3 to 57.9 ), $54.2 \%$ (49.4 to 55.9 ), and $58.5 \%$ (52.6 to 62.3 ), respectively. The median (interquartile range) R at F0, F1, F2, F3, and F4 were $89.5 \mathrm{~mm}$ (80.8 to 94.4 ), $92.3 \mathrm{~mm}$ (86.5 to 96.6), 96.9 $\mathrm{mm}$ (83.0 to 108), $101 \mathrm{~mm}$ (82.3 to 113), and $107 \mathrm{~mm}$ (99.2 to 117), respectively. The Kruskal-Wallis test revealed that there were significant differences in $\mathrm{K}_{1}(\mathrm{p}<0.01)$, MTT $(\mathrm{p}<0.001)$, and R $(\mathrm{p}<0.0001)$, depending on the stage of liver fibrosis. $V_{d}$ did not significantly depend on the stage of fibrosis $(p=0.11)$. Multiple comparisons showed significant differences $(\mathrm{p}<0.05)$ between $\mathrm{K}_{1}$ at $\mathrm{F} 0$ and at $\mathrm{F} 4$, between $\mathrm{K}_{1}$ at 
F2 and at F4, between MTT at F0 and at F4, between MTT at F1 and at F4, between MTT at F2 and at F4, between $\mathrm{R}$ at F0 and at F4, and between $\mathrm{R}$ at F1 and at F4, as Figure 2 shows.

Stepwise regression analysis showed that, of the perfusion parameters, spleen size, age, gender, MTT, R, and the presence or absence of HBV infection, and of HCV infection, were the significant explanatory variables, as Figure 3 shows. Age and gender were not added to the model because they were not significant explanatory variables. The eventual regression model was expressed by the following equation: $\mathrm{F}=$ $1.2954 *[$ presence of HBV infection $]+1.4747 *[$ presence of HCV infection $]+0.0658 *[\mathrm{MTT}]+0.0268 *[\mathrm{R}]$ -2.6065 .

The diagnostic accuracy of the combination of MTT, R, the presence or absence of HBV infection, and that of $\mathrm{HCV}$ infection, in detecting liver fibrosis was as follows: AUROC $(95 \% \mathrm{CI})=0.886(0.884-0.888)$ for $\geqq \mathrm{F} 1 ; 0.828(0.825-0.830)$ for $\geqq \mathrm{F} 2 ; 0.818(0.816-0.821)$ for $\geqq \mathrm{F} 3 ; 0.819(0.817-0.822)$ for $\mathrm{F} 4$, as Figure 4 shows.

\section{Discussion}

Our results indicated that $\mathrm{K}_{1}$ and $\mathrm{V}_{\mathrm{d}}$ did not determine the stage of fibrosis statistically. MTT in patients with cirrhosis tended to be longer than in patients with earlier stages of fibrosis. $\mathrm{R}$ in patients with intermediate fibrosis tended to be longer than in patients without fibrosis. $V_{d}$ is the apparent volume in which the contrast agent is potentially distributed throughout the extracellular space and is expressed as the product of MTT and $\mathrm{K}_{1}$. MTT tended to increase and $\mathrm{K}_{1}$ tended to decrease as a progression of liver fibrosis, so the product $\mathrm{V}_{\mathrm{d}}$ was constant and did not depend on the degree of fibrosis. That is to say, we believe that the apparent volume did not change and that blood inflow/outflow velocity decreased. In other words, the microstructures of the spleen did not change extensively in the progression of liver fibrosis, and hemodynamic change (decreasing blood inflow/outflow velocity) was more noteworthy.

The diagnostic accuracy of the combination of MTT, R, and the presence or absence of HBV infection, and that of $\mathrm{HCV}$ infection, in detecting liver fibrosis was as follows: AUROC $(95 \% \mathrm{CI})=0.828(0.825-0.830)$ for $\geqq \mathrm{F} 2 ; 0.819(0.817-0.822)$ for F4. This is not less than that of currently used noninvasive methods such 
as transient erastography ${ }^{15}$ (AUROC $=0.78$ for $\geqq \mathrm{F} 2 ; 0.89$ for $\mathrm{F} 4$ ) and serum biomarkers ${ }^{16}$ (AUROC $=$ 0.73 for $\geqq \mathrm{F} 2 ; 0.90$ for $\mathrm{F} 4$ ).

Serum fibrosis markers and liver elastography are, alone or in combination, among the most accurate noninvasive examinations for assessing the degree of liver fibrosis ${ }^{1,3}$. Indeed, these methods are highly accurate and good for fibrosis staging. However, they are less available than dynamic CT and insufficient for screening hepatocellular carcinoma, as well as for detecting complications of portal hypertension, whereas dynamic CT is widely available and sufficient for these purposes. If, in addition, dynamic CT can provide information about the degree of liver fibrosis, it is even more useful than is currently thought.

There are several studies on assessing the degree of liver fibrosis using CT perfusion of the liver ${ }^{17,18}$. However, the assessment of liver perfusion using pharmacokinetic models requires more data and higher temporal resolution, because the liver has two sources of blood (the portal vein and the hepatic artery), whereas the spleen has only one (the splenic artery). Although splenic perfusion indicates liver fibrosis only indirectly, the assessment of splenic perfusion requires less data and lower temporal resolution than the assessment of liver perfusion. Thus, splenic perfusion is easier to use than liver perfusion.

According to Ronot et al ${ }^{18}$, MTT of the liver increases in early stages of fibrosis. On the other hand, our study showed that MTT of the spleen increases in later stages of fibrosis. This may be because the liver cannot expand as the spleen does in order to compensate for portal hypertension.

Our CT protocol was for preoperative evaluation of hepatic lesions and liver vascular anatomy as well as for perfusion analysis by a 64-row detector CT scanner, so the temporal resolution in our CT protocol was lower than in usual perfusion imaging ${ }^{19-22}$. However, it was high enough to evaluate the differences among patients with different stages of liver fibrosis. This is mainly because the pharmacokinetic model of the spleen is simpler than that of the liver for evaluating perfusion parameters, as explained above.

Increased MTT of the spleen in cirrhosis may reflect dynamic changes in the spleen and in splenic 
hemodynamics. It has been demonstrated that the amount of fluid extravasation from splenic circulation increases as an adaptation to portal hypertension ${ }^{23}$. One of the main causes of portal hypertension is hyperdynamic circulation ${ }^{9,24-26}$. Once hyperdynamic circulation occurs, portal pressure increases dramatically ${ }^{27}$. It has been said that excessive systemic nitric oxide (NO) production may cause increases in splanchnic blood flow and in angiogenesis during hyperdynamic circulation ${ }^{28}$. Exactly when hyperdynamic circulation starts in the progression of liver fibrosis is not known. Morales-Ruiz et al ${ }^{29}$ showed that NO synthase was elevated in rats with cirrhosis and ascites, and Noguchi et al ${ }^{30}$ showed that there was no elevation of serum NO in patients with F4 who were Child-Pugh A and had undergone hepatectomies. Therefore, hyperdynamic circulation may start between early and advanced cirrhosis. Accordingly, increased MTT of the spleen in cirrhosis may reflect the increase in fluid extravasation from splenic circulation after hyperdynamic circulation.

Splenic blood flow increases as a progression of portal hypertension. The spleen enlarges to compensate for portal hypertension and maintain a constant splenic blood flow; MTT of the spleen does not change. However, the spleen cannot enlarge indefinitely because of anatomical limitations, so next, extravasation in the spleen occurs in order to compensate for the portal hypertension; MTT becomes longer in cirrhosis.

This study has several limitations. First, as the spleen is heterogeneously enhanced in the arterial phases, it would have been ideal to measure the whole spleen. Unfortunately, it was not practical to do so. We attempted to compensate for this by setting ROI of the spleen as large as possible. Second, we did not have enough samples to assess the difference in splenic perfusion between patients with porto-systemic collateral vessels and those without. All of the patients in our study had undergone hepatectomies, and there were not many patients with these vessels, because patients with severe cirrhosis who had developed shunts were mostly rejected for hepatectomies in preoperative consideration. Third, our surgical samples do not represent the whole liver. On the other hand, they represent a larger portion of the liver than current gold standard biopsy does. Fourth, several studies have shown that the appropriate amount of contrast agent varies with body weight ${ }^{20,31}$. We were constrained by the available data, in which the protocol was for preoperative evaluation of vascular anatomy and therefore emphasized vascular visualization (at least 600 $\mathrm{mg} \mathrm{I} / \mathrm{kg}$ is recommended as a total dose of contrast agent ${ }^{32,33}$ ). The densest contrast agent and the $100 \mathrm{~mL}$ 
dosage were adopted for purposes of preoperative evaluation.

In conclusion, the combination of spleen size and MTT, which is one of the pharmacokinetic parameters of splenic perfusion evaluated by means of dynamic $\mathrm{CT}$, is helpful in assessing the degree of liver fiborosis.

Acknowledgements The authors thank Mr. Robert Weingart for reviewing the manuscript and the radiographers at our hospital for their cooperation.

\section{Reference list}

1 Martinez SM, Crespo G, Navasa M, Forns X. Noninvasive assessment of liver fibrosis. Hepatology. 2011 Jan;53: 325-35.

2 Martin P, Lau DT, Nguyen MH, et al. A Treatment Algorithm for the Management of Chronic Hepatitis B Virus Infection in the United States: 2015 Update. Clin Gastroenterol Hepatol. 2015 Nov;13: 2071-87 e16.

3 European Association for Study of L, Asociacion Latinoamericana para el Estudio del H. EASL-ALEH Clinical Practice Guidelines: Non-invasive tests for evaluation of liver disease severity and prognosis. J Hepatol. 2015 Jul; $63:$ 237-64.

4 Rypins EB, Fajman W, Sarper R, et al. Radionuclide angiography of the liver and spleen. Noninvasive method for assessing the ratio of portal venous to total hepatic blood flow and portasystemic shunt patency. Am J Surg. 1981 Nov;142: 574-9.

5 Tsushima Y, Unno Y, Koizumi J, Kusano S. Measurement of human hepatic and splenic perfusion using dynamic computed tomography: a preliminary report. Comput Methods Programs Biomed. 1998 Aug-Sep;57: 143-6. 6 Motosugi U, Ichikawa T, Sou H, Morisaka H, Sano K, Araki T. Multi-organ perfusion CT in the abdomen using a 320-detector row CT scanner: preliminary results of perfusion changes in the liver, spleen, and pancreas of cirrhotic patients. Eur J Radiol. 2012 Oct;81: 2533-7.

$7 \quad$ Sauter AW, Feldmann S, Spira D, et al. Assessment of splenic perfusion in patients with malignant hematologic diseases and spleen involvement, liver cirrhosis and controls using volume perfusion CT (VPCT): a pilot study. Acad Radiol. 2012 May;19: 579-87.

8 Tarao K, Hoshino H, Motohashi I, et al. Changes in liver and spleen volume in alcoholic liver fibrosis of man. Hepatology. 1989 Apr;9: 589-93.

9 Mejias M, Garcia-Pras E, Gallego J, Mendez R, Bosch J, Fernandez M. Relevance of the mTOR signaling pathway in the pathophysiology of splenomegaly in rats with chronic portal hypertension. J Hepatol. 2010 Apr;52: 529-39.

10 Huang HC, Haq O, Utsumi T, et al. Intestinal and plasma VEGF levels in cirrhosis: the role of portal pressure. J Cell Mol Med. 2012 May;16: 1125-33.

11 Goshima S, Kanematsu M, Watanabe H, et al. Gd-EOB-DTPA-enhanced MR imaging: Prediction of hepatic fibrosis stages using liver contrast enhancement index and liver-to-spleen volumetric ratio. Journal of Magnetic Resonance Imaging. 2012;36: 1148-53.

12 Chen XL, Chen TW, Li ZL, et al. Spleen size measured on enhanced MRI for quantitatively staging liver fibrosis in minipigs. J Magn Reson Imaging. 2013 Sep;38: 540-7.

13 Makuuchi M, Kosuge T, Takayama T, et al. Surgery for small liver cancers. Semin Surg Oncol. 1993 Jul-Aug;9: 298-304.

14 Iida Y, Konishi J, Harioka T, Misaki T, Endo K, Torizuka K. Thyroid CT number and its relationship to iodine concentration. Radiology. 1983 Jun;147: 793-5.

15 Afdhal NH, Bacon BR, Patel K, et al. Accuracy of fibroscan, compared with histology, in analysis of liver fibrosis in patients with hepatitis B or C: a United States multicenter study. Clin Gastroenterol Hepatol. 2015 Apr;13: 772-9 e1-3.

16 Rosenberg WM, Voelker M, Thiel R, et al. Serum markers detect the presence of liver fibrosis: a cohort study. Gastroenterology. 2004 Dec;127: 1704-13.

17 Van Beers BE, Leconte I, Materne R, Smith AM, Jamart J, Horsmans Y. Hepatic perfusion parameters in chronic liver disease: dynamic CT measurements correlated with disease severity. AJR Am J Roentgenol. 2001 Mar; 176: 667-73.

18 Ronot M, Asselah T, Paradis V, et al. Liver fibrosis in chronic hepatitis C virus infection: differentiating minimal from intermediate fibrosis with perfusion CT. Radiology. 2010 Jul;256: 135-42. 
19

Prezzi D, Khan A, Goh V. Perfusion CT imaging of treatment response in oncology. Eur J Radiol. 2015

Mar 27.

20 Miles KA, Lee TY, Goh V, et al. Current status and guidelines for the assessment of tumour vascular support with dynamic contrast-enhanced computed tomography. Eur Radiol. 2012 Jul;22: 1430-41.

21 Kim SH, Kamaya A, Willmann JK. CT perfusion of the liver: principles and applications in oncology. Radiology. 2014 Aug;272: 322-44.

22 Fischer MA, Kartalis N, Grigoriadis A, et al. Perfusion computed tomography for detection of hepatocellular carcinoma in patients with liver cirrhosis. Eur Radiol. 2015 Nov;25: 3123-32.

23 Kaufman S, Levasseur J. Effect of portal hypertension on splenic blood flow, intrasplenic extravasation and systemic blood pressure. Am J Physiol Regul Integr Comp Physiol. 2003 Jun;284: R1580-5.

24 Bolognesi M, Merkel C, Sacerdoti D, Nava V, Gatta A. Role of spleen enlargement in cirrhosis with portal hypertension. Dig Liver Dis. 2002 Feb;34: 144-50.

25 Iwakiri Y. Pathophysiology of portal hypertension. Clin Liver Dis. 2014 May; 18: 281-91.

26 Fernandez M. Molecular pathophysiology of portal hypertension. Hepatology. 2015 Apr;61: 1406-15.

27 Iwakiri Y, Groszmann RJ. The hyperdynamic circulation of chronic liver diseases: from the patient to the molecule. Hepatology. 2006 Feb;43: S121-31.

28 Sumanovski LT, Battegay E, Stumm M, van der Kooij M, Sieber CC. Increased angiogenesis in portal hypertensive rats: role of nitric oxide. Hepatology. 1999 Apr;29: 1044-9.

29 Morales-Ruiz M, Jimenez W, Perez-Sala D, et al. Increased nitric oxide synthase expression in arterial vessels of cirrhotic rats with ascites. Hepatology. 1996 Dec;24: 1481-6.

30 Noguchi N, Teramoto K, Ochiai T, et al. The close participation of ET-1 and NO in human hepatic hemodynamics. Hepatol Res. 2006 Oct;36: 86-93.

31 Heiken JP, Brink JA, McClennan BL, Sagel SS, Crowe TM, Gaines MV. Dynamic incremental CT: effect of volume and concentration of contrast material and patient weight on hepatic enhancement. Radiology. 1995 May;195: 353-7.

32 Yamashita Y, Komohara Y, Takahashi M, et al. Abdominal helical CT: evaluation of optimal doses of intravenous contrast material--a prospective randomized study. Radiology. 2000 Sep;216: 718-23.

33 Yanaga Y, Awai K, Nakaura T, et al. Optimal contrast dose for depiction of hypervascular hepatocellular carcinoma at dynamic CT using 64-MDCT. AJR Am J Roentgenol. 2008 Apr;190: 1003-9.

\section{Table and Figure legends:}

\section{Table 1}

Patient characteristics

133 consecutive patients (2008-2013) who had undergone dynamic CT within 30 days before hepatectomy

Characteristic

Gender (men/women)

Age (years)

Interval between CT and hepatectomy (days)

Long axis of spleen $(\mathrm{mm})$

Stage of fibrosis (no. of patients)

F0 (absent fibrosis)

F1 (portal fibrosis)

F2 (portal fibrosis with few septa)

F3 (septal fibrosis)

F4 (cirrhosis)

\section{Datum}

$87 / 46$

$68(37-83) *$

$14(1-29)^{*}$

$94(65-149)^{*}$

17

30

16

27

*: Median (range) 


\section{Table 2}

The protocol of dynamic contrast-enhanced CT

$\begin{array}{lc}\begin{array}{l}\text { 64-row CT scanner } \\ \text { Range of scan }\end{array} & \text { Caudal } 25 \mathrm{~cm} \text { from upper diaphragm } \\ \text { Size of collimation } & 0.625 \mathrm{~mm} \\ \text { Thickness of reconstruction } & 2.5 \mathrm{~mm} \\ \text { Number of scans } & 10 \text { phases } \\ \text { Timing of scans } & \text { Before injection and at 22, 28, 34, 40, 46, } \\ & 52,58,90, \text { and } 210 \text { (s) after injection } \\ & 7 \text { arterial phases were scanned during one } \\ & \text { breath hold with oxygen administration } \\ \text { Effective radiation dose } & 48.9 \mathrm{mSv}(48.2-48.9)^{*} \\ \text { Contrast agent } & 370 \mathrm{mg} / \mathrm{mL} \\ \text { Concentration of iodine } & 100 \mathrm{~mL} \\ \text { Total dose } & 3 \mathrm{~mL} / \mathrm{s} \\ \text { Rate of injection } & \text { 22-gauge catheter in median cubital vein }\end{array}$

*: Median (interquartile range)

\section{Figure 1}

Method of setting ROIs

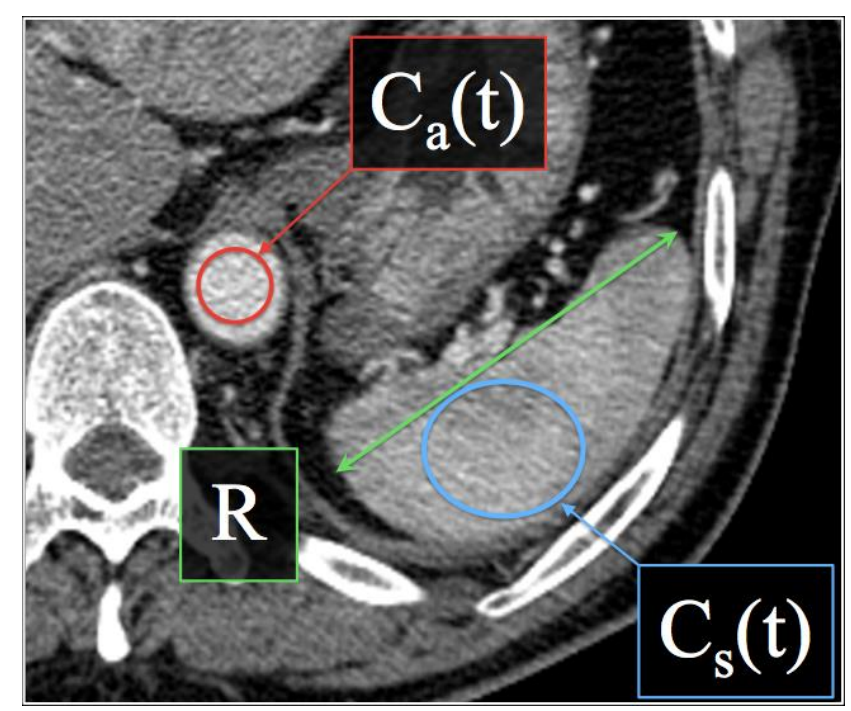

Two readers set elliptical ROIs, as large as possible, located at the spleen $\left[\mathrm{C}_{\mathrm{s}}(\mathrm{t})\right]$ and at the aorta $\left[\mathrm{C}_{\mathrm{a}}(\mathrm{t})\right]$ in the same slice, and measured the long axis of the spleen (R) in each of 133 cases. 


\section{Figure 2}

Statistical analysis of liver fibrosis stages (F0-4) vs. splenic perfusion parameters and spleen size
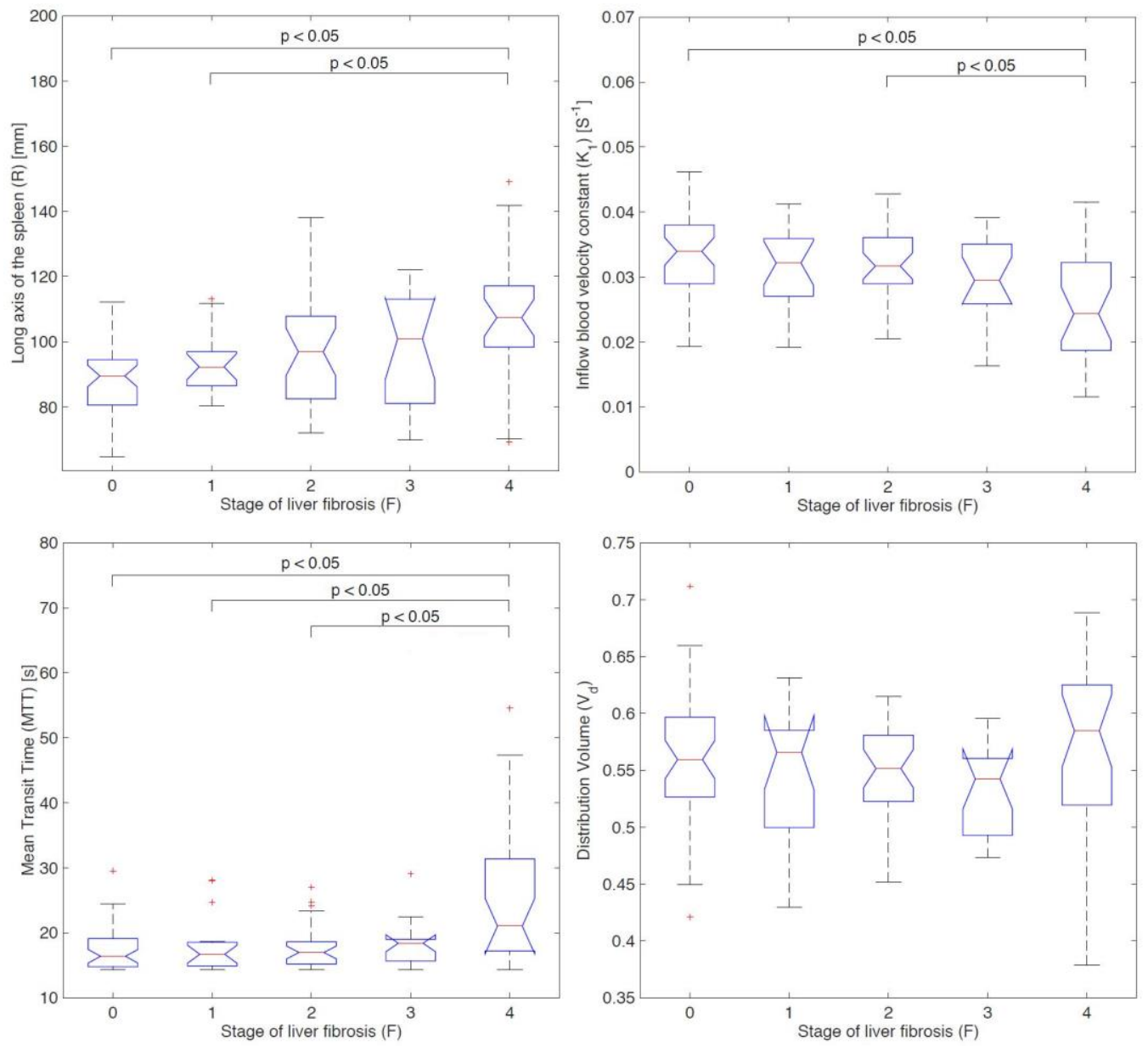

We plotted splenic perfusion parameters and spleen size against stage of liver fibrosis in 133 cases. 
Figure 3

Results of stepwise regression analysis

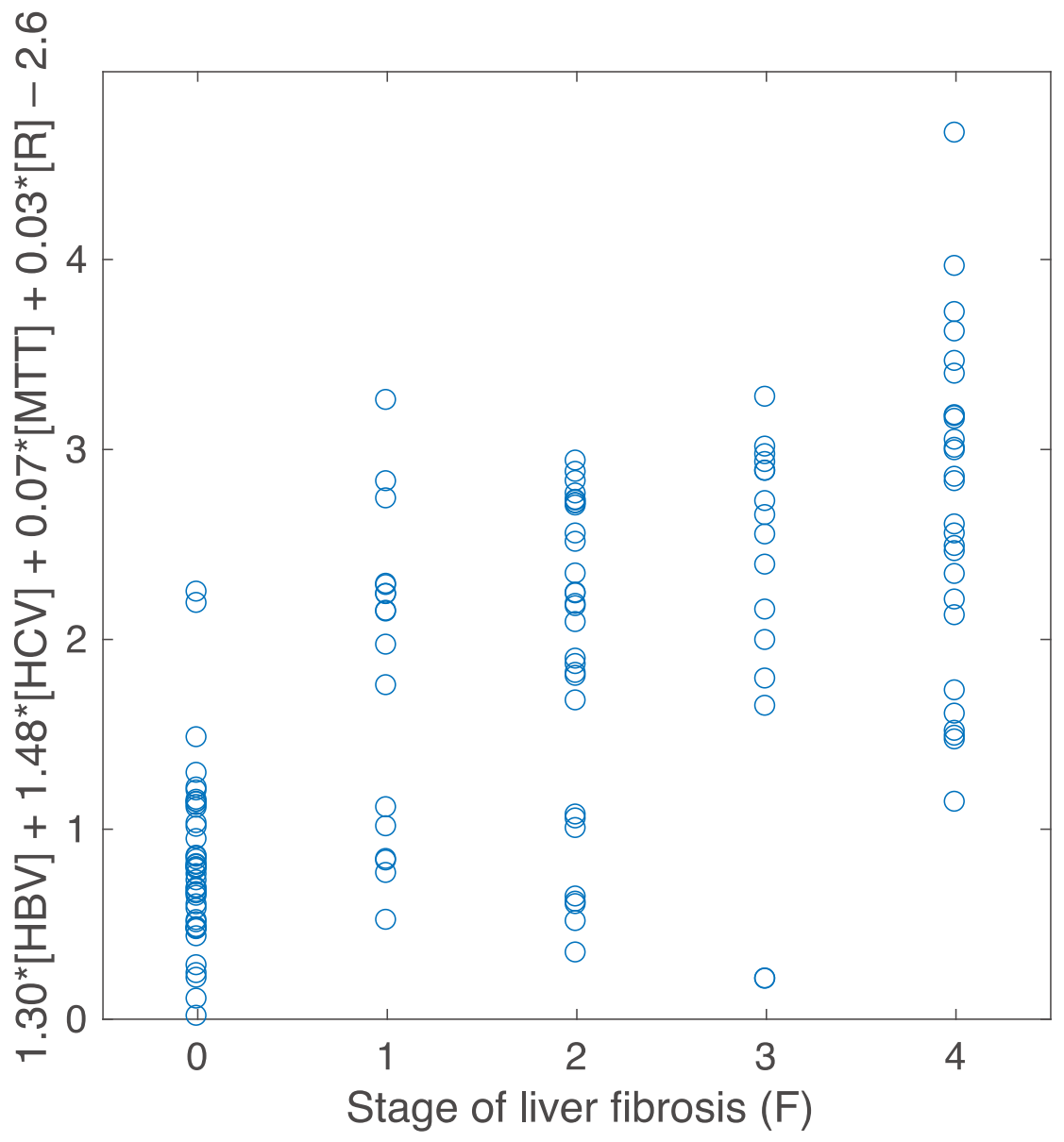

We plotted the stage of liver fibrosis indicated by the equation combining MTT, R, and the presence or absence of HBV infection, and that of HCV infection against the actual stage for each patient. 
Figure 4

ROC curves for the equation combining MTT, R, and the presence or absence of HBV infection, and that of HCV infection

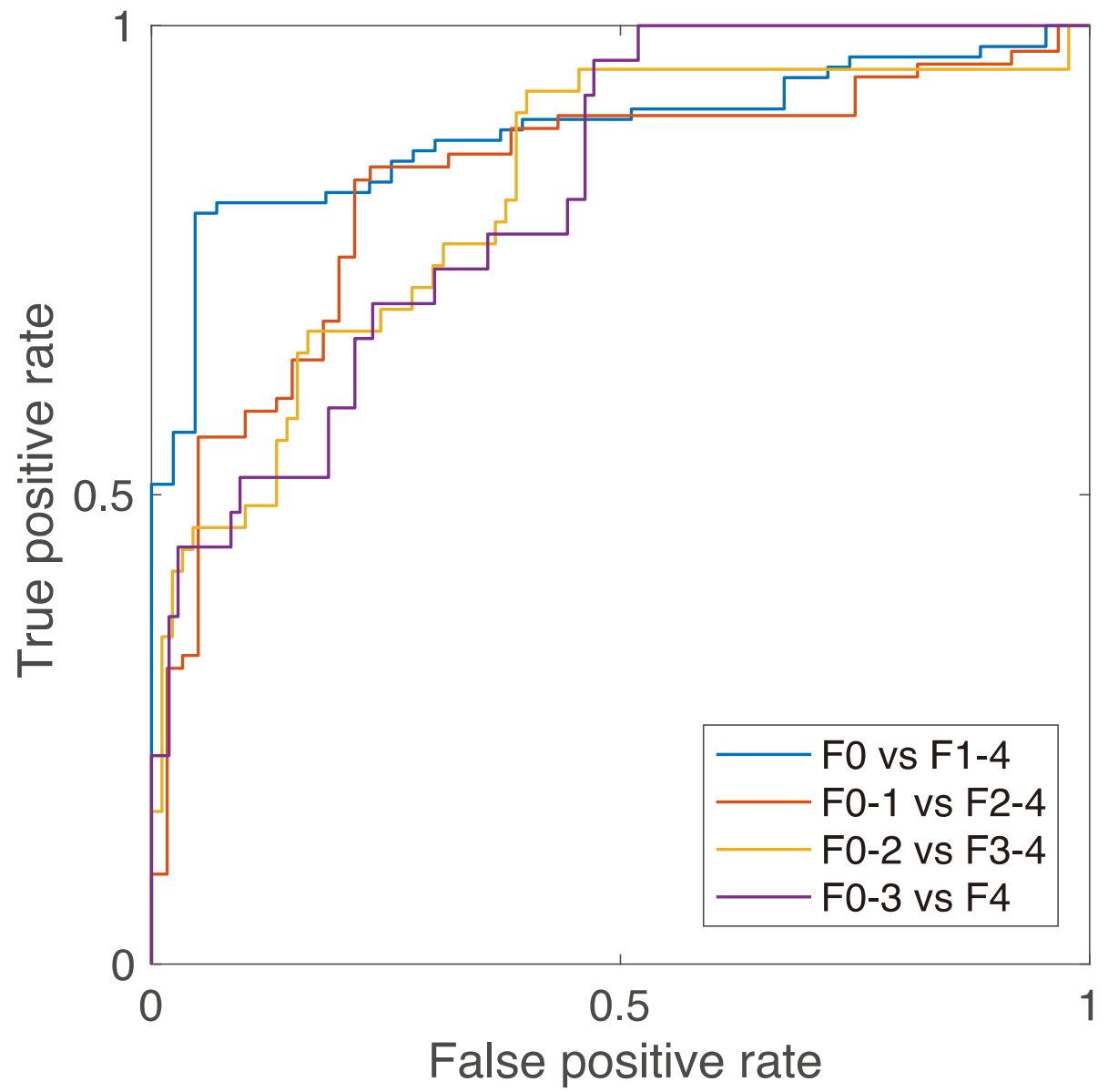

The ROC curves are shown for the reliability of the equation combining MTT, R, and the presence or absence of $\mathrm{HBV}$ infection, and that of $\mathrm{HCV}$ infection for discriminating between $\mathrm{FO}$ and F1-4, between F01 and F2-4, between F0-2 and F3-4, and between F0-3 and F4. 